\title{
Epithelial-mesenchymal transition in tumor metastasis: a method to the madness
}
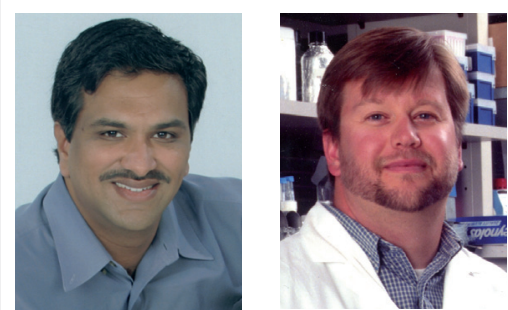

\author{
"Dissemination of cancer is not \\ simply a random dispersion of \\ cells, but instead represents an \\ ordered and systematic method \\ to this madness."
}

Venkateshwar G Keshamounit \& William P Schiemann

†Author for correspondence: Division of Pulmonary and Critical Care Medicine, Department of Internal Medicine, University of Michigan Medical Center, 4062 BSRB, 109 Zina Pitcher Place, Ann Arbor, MI 48109, USA = Tel.: +17346479527 =. Fax: +17346152331=vkeshamo@med.umich.edu

Were it not for the ability of carcinoma cells to metastasize and colonize distant organs, all solid tumors would present medically as a group of chronic but manageable diseases. There has been significant progress in the understanding of how cancer cells acquire five of the six essential hallmarks proposed for their transformation [1]. Unfortunately, it still remains unclear as to how and when cancer cells acquire the ability to metastasize - that is, the sixth and final hallmark that is responsible for more than $90 \%$ of cancer-related mortality [1]. However, it has long been recognized that the dissemination of cancer is not simply a random dispersion of cells, but instead represents an ordered and systematic method to this madness. Indeed, epithelial-mesenchymal transition (EMT) is one such method that has been proposed to initiate the metastasis of carcinoma cells [2].

Epithelial-mesenchymal transition was first recognized as a conserved embryonic and developmental process that facilitates the dispersion of cells that ultimately leads to the generation of distinct tissue types [3]. In undergoing EMT, cells lose their epithelial properties, while acquiring mesenchymal properties that enable transitioned cells to migrate to predetermined destinations [4]. The idea that a similar process is reactivated during tumor progression and other pathologies, including wound healing, tissue regeneration and organ fibrosis, has gained significant ground and acceptance in recent years. Indeed, this fact is readily apparent in the sheer number of publications on this topic, and in the number of EMTfocused sessions and dedicated meetings that have grown exponentially in the last few years. It is now widely accepted that EMT plays an important role during tumor progression and confers certain fundamental abilities to cancer cells that are essential for tumor metastasis. These include the ability to migrate, resist anoikis and induce immunosuppression [5-7].

The precise contribution of EMT to tumor metastasis is still a subject of considerable debate in the scientific literature [8]. Recent reports of EMT in in vivo animal models and human studies [9-12], to a certain degree, have softened the arguments for lack of concrete in vivo evidence. However, convincing demonstration of a true phenotypic switch is still yet to come. The other dismissive argument that EMT is simply reflective of genomic instability in cancer cells is also fading in light of increasing numbers of studies reporting EMT that occurs in normal epithelial cells from various organs in response to injury $[9,11,13]$.

Reports of EMT conferring resistance to certain classes of drugs and therapeutic modalities, and correlation of EMT gene signatures with poor outcomes have been described [14-16]. These observations, together with the recent finding that EMT may confer stem cell-like properties to resulting mesenchymal cells [17] have highlighted the clinical relevance of this process. Consequently, several groups, both in industry and academia, are actively pursuing the discovery of novel molecules to target EMT [18].

“... any effort to identify context-specific signals should consider the physiological state of the epithelium in which EMT is

taking place - that is, whether it transpires in normal, transformed or injured epithelium..."

Recently, Kalluri and Weinberg proposed to classify EMT into three distinct subtypes based on the biological context in which they

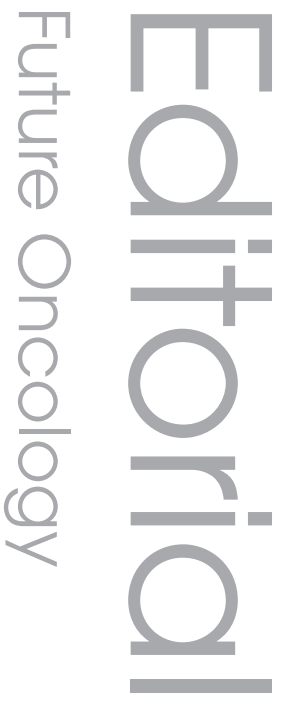


occur [4]. This new terminology was not available at the time the reviews for this special focus issue were accepted for publication, and as such, this classification is not used herein. With the exception of the review by Micalizzi et al. [19], the other articles have predominantly discussed what now could be referred to as type III EMT in the new classification system, which is EMT in the context of tumor progression. By contrast, the article by Micalizzi et al. describes the regulators of developmental EMT, which now is known as type I EMT in the new classification scheme, and discusses the transcriptional reactivation of type I EMT in the context of type III EMT. Particularly interesting is the discussion of their own work investigating the role of two new players, Six1 and Six4, in the EMT of mouse mammary tumors. Radaelli et al. provide a very elegant historical perspective by discussing some of the early descriptions of EMT in mouse tumors [20], some of which date as far back as the year 1854. They also present an interesting comparison of EMT in mouse and human pathologies. A very comprehensive review of the regulatory pathways implicated in TGF- $\beta$-induced EMT in normal and malignant cells of the breast is provided in the article by Wendt et al. [21], and finally, van Zijl et al. [22] review the evidence for EMT in hepatocellular carcinoma and discussed its implications for the treatment of these tumors.

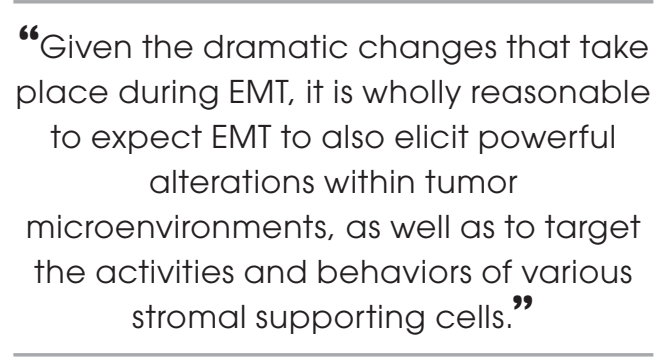

Pathways and molecules that distinguish EMT in tumor progression from the other two biological contexts are far from clear. However, any effort to identify context-specific signals should consider the physiological state of the epithelium in which EMT is taking place that is, whether it transpires in normal, transformed or injured epithelium, and how these unique epithelial states impact the functional consequences of the resulting EMT. Indeed, the vast majority of EMT studies to date have solely focused on assessing the functional consequences of EMT in solely altering the behaviors and functions of tumor cells, not their accompanying stromal components. Given the dramatic changes that take place during EMT, it is wholly reasonable to expect EMT to also elicit powerful alterations within tumor microenvironments, as well as to target the activities and behaviors of various stromal supporting cells. Therefore, the implications of EMT on the interactions of tumor cells with their accompanying stromal and microenvironmental components clearly need to be explored in future studies.

Financial \& competing interests disclosure
The research work in Keshamouni's laboratory is sup-
ported by grants from Flight Attendant Medical Research
Institute, NIH (CA132571), and American Cancer
Society (CSM-116801). Dr Schiemann is supported in
part by grants from the NIH (CA114039 and
CA129359), the DOD (BC084651) and the Komen
Foundation (BCTR0706967). The authors have no
other relevant affiliations or financial involvement with
any organization or entity with a financial interest in
or financial conflict with the subject matter or materials
discussed in the manuscript apart from those disclosed.
No writing assistance was utilized in the production
of this manuscript.

Financial \& competing interests disclosure

The research work in Keshamouni's laboratory is supported by grants from Flight Attendant Medical Research Institute, NIH (CA132571), and American Cancer Society (CSM-116801). Dr Schiemann is supported in 4039 and $C A 129359)$, the DOD (BC084651) and the Komen other relevant affliations or financial involvement with any organization or entity with a financial interest in or financial conflict with the subject matter or materials discussed in the manuscript apart from those disclosed. of this manuscript.

\section{Bibliography}

Papers of special note have been highlighted as:

- of interest

-. of considerable interest

1. Hanahan D, Weinberg RA: The hallmarks of cancer. Cell 100, 57-70 (2000).

-. Seminal review describing six hallmarks of a cancer cell.

2. Thiery JP: Epithelial-mesenchymal transitions in tumour progression. Nat. Rev. Cancer 2, 442-454. (2002).

3. Acloque H, Adams MS, Fishwick K, Bronner-Fraser M, Nieto MA: Epithelial-mesenchymal transitions: the importance of changing cell state in development and disease. J. Clin. Invest. 119, 1438-1449 (2009).

4. Kalluri R, Weinberg RA: The basics of epithelial-mesenchymal transition. J. Clin. Invest. 119, 1420-1428 (2009).

- Proposed classification of the epithelial-mesenchymal transition (EMT) into three subtypes.

5. Yang J, Weinberg RA: Epithelial-mesenchymal transition: at the crossroads of development and tumor metastasis. Dev. Cell 14, 818-829 (2008).

6. Thiery JP, Sleeman JP: Complex networks orchestrate epithelial-mesenchymal transitions. Nat. Rev. Mol. Cell Biol. 7, 131-142 (2006).

7. Kudo-Saito C, Shirako H, Takeuchi T et al.: Cancer metastasis is accelerated through immunosuppression during Snail-induced EMT of cancer cells. Cancer Cell. 15, 195-206 (2009).

- First paper to demonstrate that EMT also induces immunosuppression, along with enhanced invasion, to promote metastasis.

8. Tarin D, Thompson EW, Newgreen DF: The fallacy of epithelial mesenchymal transition in neoplasia. Cancer Res. 65 , 5996-6000; discussion 6000-5991 (2005). 
9. Kim KK, Kugler MC, Wolters PJ et al.: Alveolar epithelial cell mesenchymal transition develops in vivo during pulmonary fibrosis and is regulated by the extracellular matrix. Proc. Natl Acad. Sci. USA 103, 13180-13185 (2006).

- First paper to demonstrate in vivo EMT in the context of tissue fibrosis by tracking cell fate.

10. Trimboli AJ, Fukino K, de Bruin A et al:: Direct evidence for epithelial-mesenchymal transitions in breast cancer. Cancer Res. 68, 937-945 (2008).

- First in vivo evidence of EMT in the context of tumor progression in a mouse model of breast cancer.

11. Zeisberg M, Yang C, Martino M et al: Fibroblasts derive from hepatocytes in liver fibrosis via epithelial to mesenchymal transition. J. Biol. Chem. 282, 23337-23347 (2007).

12. Reckamp KL, Gardner BK, Figlin RA et al.: Tumor response to combination celecoxib and erlotinib therapy in non-small cell lung cancer is associated with a low baseline matrix metalloproteinase- 9 and a decline in serum-soluble e-cadherin. J. Thorac. Oncol. 3, $117-124$ (2008).

- First correlation of EMT markers to therapeutic response in patients with lung cancer.

13. Iwano M, Plieth D, Danoff TM, Xue C, Okada H, Neilson EG: Evidence that fibroblasts derive from epithelium during tissue fibrosis. J. Clin. Invest. 110, 341-350 (2002).
- First paper to demonstrate contribution of EMT to tissue fibrosis.

14. Dimeo TA, Anderson K, Phadke P et al.: Anovel lung metastasis signature links wnt signaling with cancer cell self-renewal and epithelial-mesenchymal transition in basal-like breast cancer. Cancer Res. 69(13), 5364-5373 (2009).

15. Singh A, Greninger P, Rhodes D et al.: A gene expression signature associated with 'K-ras addiction' Reveals regulators of EMT and tumor cell survival. Cancer Cell 15, 489-500 (2009).

16. Thomson S, Buck E, Petti F et al.: Epithelial to mesenchymal transition is a determinant of sensitivity of non-small-cell lung carcinoma cell lines and xenografts to epidermal non receptor inhibition. Cancer Res. 65, 9455-9462 (2005).

17. Mani SA, Guo W, Liao MJ et al.: The epithelial-mesenchymal transition generates cells with properties of stem cells. Cell 133, 704-715 (2008).

- First paper to demonstrate a link between EMT and stemness.

18. Lee JM, Dedhar S, Kalluri R, Thompson EW: The epithelialmesenchymal transition: new insights in signaling, development, and disease. J. Cell Biol. 172, 973-981 (2006).

19. Micalizzi DS, Ford HL: Epithelial-mesenchymal transition in development and cancer. Future Oncol. 5(8), 1129-1143 (2009).
20. Radaelli E, Damonte P, Cardiff RD: Epithelial-mesenchymal transition in mouse mammary tumorigenesis. Future Oncol. 5(8), 1113-1127 (2009).

21. Wendt MK, Allington TM, Schiemann WP: Mechanisms of epithelial-mesenchymal transition by TGF- $\beta$. Future Oncol. 5(8), 1145-1168 (2009)

22. van Zijl F, Zulehner G, Petz M et al.: Epithelial-mesenchymal transition in hepatocellular carcinoma. Future Oncol. 5(8), 1169-1179 (2009).

\section{Affiliations}

- Venkateshwar G Keshamouni Division of Pulmonary and Critical Care, Department of Internal Medicine, University of Michigan Medical Center, Ann Arbor, MI 48109, USA

Tel.: +1 7346479527

Fax: +17346152331

vkeshamo@med.umich.edu

- William P Schiemann Department of Pharmacology, University of Colorado Denver, Anschutz Medical Campus, Aurora, CO 80045, USA 